\title{
Dietas Hiperglicídicas: Efeitos da Substituição Isoenergética de Gordura por Carboidratos Sobre o Metabolismo de Lipídios, Adiposidade Corporal e Sua Associaçāo com Atividade Física e com o Risco de Doença Cardiovascular
}

\begin{abstract}
RESUMO
Há vários anos, vem sendo recomendada à população em geral a diminuição da gordura dietética, a fim de se prevenir doenças cardiovasculares, obesidade, diabetes mellitus tipo 2 , dentre outras doenças crônicas. A conseqüência desta recomendação tem sido a substituição de gorduras por carboidratos, resultando na adoção de dietas hiperglicídicas. Porém, ainda é muito discutido se dietas hiperglicídicas devem ser recomendadas à população geral. Pesquisas apontam controvérsias a respeito da associação entre este tipo de dieta e efeitos deletérios sobre a saúde e aspectos metabólicos, tais como aumento da lipogênese de novo, aumento da trigliceridemia, diminuição das concentrações de HDL e efeitos sobre a adiposidade. Esta revisão aborda os efeitos de mudanças na composição dietética, particularmente da dieta hiperglicídica, sobre fatores de risco para doenças cardiovasculares, tais como dislipidemia, e obesidade. Revisa, também, sua interação com a atividade física, uma vez que ainda não se sabe com que extensão ela pode minimizar possíveis efeitos deletérios de dietas hiperglicídicas em longo prazo. (Arq Bras Endocrinol Metab 2007;51/3:389-400)
\end{abstract}

Descritores: Dietas hiperglicídicas; Composição da dieta; Dislipidemias; Lipogênese de novo; Adiposidade; Atividade física

\section{ABSTRACT}

High-Carbohydrate Diets: Effects on Lipid Metabolism, Body Adiposity and Its Association With Physical Activity and Cardiovascular Disease Risk.

For many years the reduction in the dietary fat has been recommended to the population, in order to prevent cardiovascular diseases, obesity, type 2 diabetes mellitus, among other chronic diseases. The consequence has been the replacement of carbohydrates by fat, resulting in the adoption of high carbohydrate diets. However, it has been still discussed if very rich carbohydrate diets should be recommended to the general population. Researches point out controversies about the association between these dietary habits and harmful effects on health and metabolic aspects, such as raise in de novo lipogenesis and triglyceride concentration, reduction in HDL concentration and effects on adiposity. This review evaluates the effects of diet modification, particularly the high-carbohydrate diet, in cardiovascular risk factors such as dyslipidemia and obesity. It also reviews its interaction with physical activity since it is still unknown with which extension it can minimize possible harmful effects of high carbohydrate diets in the long term. (Arq Bras Endocrinol Metab 2007;51/3:389-400)

Keywords: High carbohydrate diets; Diet composition; Dyslipidemia; de novo lipogenesis; Adiposity; Physical activity revisão

\author{
Viviane O. Polacow \\ ANTONIO H. LANCHA JUNIOR
}

Departamento de Biodinâmica do Movimento do Corpo Humano, Escola de Educação Física e Esporte, Universidade de São Paulo, SP.

Recebido em 08/04/05

Revisado em 12/07/06 e 06/09/06 Aceito em 11/10/06 
$\mathrm{H}$ Á MUITOS ANOS, PESQUISADORES de todo o mundo têm procurado fazer associações entre padrões alimentares e riscos de morbidade e mortalidade. Após o fim da Segunda Guerra Mundial, notou-se, nos Estados Unidos, grande aumento na incidência de doenças cardiovasculares e, a partir dos anos 50, pesquisas indicaram pela primeira vez a associação entre concentrações de colesterol sérico e problemas cardíacos (1). Keys e cols. (2) publicaram, na década de 1950, o primeiro artigo que fornecia recursos quantitativos para a predição das respostas do colesterol sérico a lipídios da dieta. Surgiram, então, as primeiras recomendações, por parte de cientistas e da American Heart Association (AHA), para diminuição da ingestão de gordura, principalmente a saturada, para homens com níveis de colesterol altos.

A partir daí, alimentação saudável nos Estados Unidos, e praticamente em todo o mundo, tem sido considerada dieta hipolipídica (3). Aos olhos do público em geral, gordura tornou-se sinônimo de calorias e, freqüentemente, considera-se erroneamente que alimentos low-fat (pobres em gorduras) não são capazes de gerar acúmulo de gordura corporal (4). Contudo, os produtos low-fat disponíveis no mercado podem confundir os consumidores uma vez que, muitas vezes, contêm grande concentração de açúcar e amido, além de alta densidade energética (4).

Controvérsias à parte, a fabricação de produtos alimentícios com teor reduzido de gordura tornou-se um grande negócio; a indústria investe bilhões de dólares anualmente em pesquisas para o desenvolvimento e marketing de substitutos de gordura palatáveis, vendendo a mensagem de que menos gordura significa saúde. Nos Estados Unidos, existem inúmeras políticas públicas no sentido de incentivar a redução do consumo de lipídios, na tentativa de deter o avanço da epidemia de obesidade que acomete aquele país, além de outros países industrializados (5), mas que também começa a afetar países em desenvolvimento, como o Brasil (6).

O USDA (Departamento de Agricultura dos Estados Unidos) recomenda, por meio da pirâmide alimentar (7), que gordura e óleos sejam usados com moderação e sua proporção na dieta não deve ultrapassar 30\% do valor energético total (VET). Já o mais recente Dietary Guidelines for Americans (Guia alimentar para americanos) (8) recomenda que consumo de lipídios esteja entre 20 e 35\% do VET, principalmente na forma de óleos vegetais, nozes e peixes. O guia alimentar para a população brasileira recomenda que o consumo de lipídios esteja entre 15\% e 30\% do VET (9), recomendação semelhante à da Organização Mundial de Saúde (10). A AHA (11) recomenda para a população em geral a diminuição da gordura saturada da dieta para menos do que $10 \%$ do VET da dieta. Alguns autores, como Ornish (12), vão além e defendem que benefícios máximos para a saúde seriam atingidos com dietas contendo somente $10 \%$ de energia na forma de lipídios totais.

Todas as recomendações mencionadas baseiamse em dados experimentais ou, especialmente, em estudos epidemiológicos. Contudo, estudos epidemiológicos transversais podem detectar a associação entre duas variáveis, porém não podem provar relação de causa e efeito. Isso é especialmente verdadeiro quando existem outros fatores envolvidos, os quais podem afetar esta associação, como, por exemplo, quando se correlaciona a ingestão excessiva de gordura com o aumento da prevalência de obesidade, mas não se leva em consideração a avaliação da prática de atividade física dos indivíduos. Assim sendo, muita controvérsia surgiu a respeito da relação entre ingestão de gordura e riscos para a saúde. Após décadas de pesquisas, ainda é debatida a proposição de que dietas hipolipídicas beneficiariam, com caráter preventivo, indivíduos saudáveis que ainda não apresentam fatores de risco para doenças cardiovasculares (3).

Dados epidemiológicos da população americana ilustram bem esta divergência: desde a década de 1970, apesar da queda no consumo médio de gorduras de $40 \%$ para $34 \%$ do VET, com concomitante diminuição das mortes por doenças cardíacas, o número de procedimentos médicos para tratamento destas aumentou de 1,2 para 5,4 milhões (3). Isto significa que não houve diminuição na incidência das doenças cardiovasculares, mas, sim, avanços na área médica, tais como métodos mais invasivos e de maior sensibilidade diagnóstica, que tornaram possíveis a detecção de mais casos, mais precocemente, o que pode ter interferido no quadro de mortalidade.

Contudo, mesmo a redução na ingestão de gorduras por parte dos americanos ainda é questionável. Vieses nas avaliações de consumo alimentar são muito comuns. Por exemplo, o sub-relato da ingestão alimentar, de alimentos ricos em gordura em especial, que envolve principalmente indivíduos alertados freqüentemente sobre os efeitos deletérios de uma dieta hiperlipídica, pode fazer com que estes relatem sua ingestão de acordo com o mais desejável, mas não necessariamente o real (13). O sub-relato seletivo da ingestão alimentar também ocorre constantemente em avaliações do consumo alimentar de indivíduos obesos ou que querem perder peso (14).

Da mesma forma, já é conhecida a divergência a respeito da associação entre acúmulo de gordura 
corporal e o consumo de lipídios $(15,16)$. Uma vez que a obesidade é um forte fator de risco, não só para doenças cardiovasculares mas também para dislipidemias, diabetes mellitus tipo 2 , hipertensão, osteoartrite, apnéia do sono, alguns tipos de câncer, entre outros problemas de saúde (17), procura-se cada vez mais descobrir padrões alimentares que predisponham ao ganho de gordura corporal, a fim de se implantar políticas de saúde pública de prevenção do problema.

A prevalência de obesidade nos Estados Unidos aumentou vertiginosamente nos últimos anos, assim como a prevalência de diabetes mellitus tipo $2(18,19)$. Como a obesidade e o diabetes mellitus tipo 2 são fatores de risco para doenças cardiovasculares, pode-se explicar por que a incidência das últimas não está diminuindo na velocidade esperada. Curiosamente, este fato coincide com o surgimento das recomendações para redução no consumo de gorduras por parte do governo norte-americano. Ou seja, essas doenças tiveram sua prevalência aumentada justamente no momento em que se estimulou a redução do consumo de gorduras. No entanto, segundo Astrup (20), este "paradoxo americano" pode ser facilmente explicado pela concomitante redução do nível de atividade física, que diminuiu as necessidades de ingestão calórica total e, conseqüentemente, de gorduras.

Apesar das recomendações para adoção de dietas hipolipídicas, a manipulação dietética não é tão simples. Diminuindo-se o conteúdo de algum nutriente específico, altera-se toda a composição da dieta. Dietas hipolipídicas, contendo menos do que $30 \%$ do VET na forma de lipídios, são, freqüentemente, hiperglicídicas. Enquanto uma restrição moderada em lipídios (25 a 30\% do VET) pode resultar em uma dieta normoglicídica, contendo cerca de $55 \%$ do VET na forma de carboidratos, restrições mais severas (10 a 15\% do VET na forma de lipídios) resulta em dietas hiperglicídicas (mais do que $60 \%$ do VET na forma de carboidratos). O guia alimentar para a população brasileira recomenda que o consumo de carboidratos fique entre 55 e $75 \%$ do VET (9), e o guia alimentar para americanos, entre 45 e 65\% (8). Desta forma, nota-se que, mesmo estando de acordo com diversos órgãos de saúde, dietas normoglicídicas e hiperglicídicas encontram-se nos extremos de uma mesma recomendação, a de diminuir a quantidade de lipídios da dieta, e têm conseqüências distintas sobre o metabolismo e armazenamento tanto de carboidratos quanto de lipídios (21). A principal crítica feita às recomendações para adoção de dietas hipolipídicas, portanto, é a de que estas encorajariam uma mudança alimentar para dietas muito ricas em carboidratos, os quais acabam sendo principalmente aqueles provenientes de alimentos processados e com alto índice glicêmico, que podem apresentar efeitos adversos graves à saúde, entre os quais estão: hipertrigliceridemia e diminuição na concentração plasmática de HDLcolesterol $(8,11,22)$ e aumento da adiposidade $(21)$.

Uma das principais questões ainda não resolvidas no campo da nutrição diz respeito ao consumo ideal de gorduras em relação aos outros macronutrientes, particularmente os carboidratos (23). Uma importante questão levantada é se a composição da dieta, mais especificamente a razão lipídios / carboidratos, afeta os fatores de risco para a síndrome metabólica, independentemente da obesidade (23).

Praticamente todas as estratégias nutricionais bem aceitas pela comunidade científica, e que visam a redução dos riscos de doenças cardiovasculares e da síndrome metabólica, pregam a diminuição da proporção de gordura saturada da dieta, uma vez que este é o tipo de gordura dietética que está mais fortemente relacionado a risco cardiovascular (24). Duas estratégias nutricionais são comumente utilizadas e recomendadas para prevenir e combater os riscos de doenças cardiovasculares e a síndrome metabólica, sendo que ambas dizem respeito à composição da dieta (23). A primeira é a dieta hipolipídica e, conseqüentemente, rica em carboidratos, que apresenta no máximo 20\% do VET correspondendo a lipídios e cerca de $65 \%$ do VET correspondendo a carboidratos. A segunda é uma dieta com conteúdo mais alto de lipídios (cerca de $35 \%$ a $40 \%$ do VET), rica em ácidos graxos monoinsaturados e tradicionalmente chamada de dieta mediterrânea (23). Em ambas as dietas, ácidos graxos com poder de elevar a colesterolemia, como os ácidos graxos saturados, contribuem com somente cerca de 7\% do VET.

A questão que ganha destaque, portanto, é: qual nutriente deve substituir a gordura saturada? A substituição isoenergética por carboidratos resultaria em uma dieta hipolipídica e hiperglicídica. Já a substituição por lipídios ricos em ácidos graxos monoinsaturados configuraria uma dieta semelhante à dieta mediterrânea, e é defendida por alguns pesquisadores $(4,25)$, que consideram a substituição isoenergética de gorduras por carboidratos um risco à saúde.

Ainda existe muita polêmica a respeito de qual deve ser a composição ideal da dieta da população em geral. Não há consenso a respeito da capacidade que dietas ricas em carboidratos teriam na prevenção de doenças cardiovasculares e obesidade. Ainda não é sabido, tampouco, quanto a substituição de gorduras por carboidratos pode trazer de benefícios e até que 
ponto se pode restringir a ingestão de lipídios, aumentando a ingestão de carboidratos, sem causar efeitos adversos (no perfil lipídico e na adiposidade corporal, por exemplo).

A seguir, é feita uma revisão que aborda alguns pontos relacionados às conseqüências de dietas hiperglicídicas e de alterações na composição das dietas na saúde e em certos aspectos metabólicos, assim como sua associação com a atividade física.

\section{DIETAS HIPERGLICÍDICAS}

\section{Conseqüências sobre 0 metabolismo lipídico}

O processo biológico pelo qual carbonos provenientes de carboidratos (glicose, frutose, lactose etc.) são transformados em lipídios (ácidos graxos) é chamado de lipogênese de novo (26). A importância deste processo é seu possível papel no desenvolvimento de dislipidemias e no acúmulo de gordura corporal.

Embora a lipogênese de novo seja altamente ativa em roedores, sua contribuição para o fluxo de ácidos graxos em humanos ainda não foi bem definida devido à falta de métodos capazes de mensurar a síntese de lipídios in vivo em humanos (26). Recentemente, métodos para mensurar a lipogênese de novo em humanos foram desenvolvidos (27), os quais podem fornecer ferramentas úteis para futuros estudos sobre o tema.

Fatores nutricionais podem afetar a expressão das enzimas que participam da lipogênese de novo, como a acetil-CoA carboxilase (ACC), que catalisa a carboxilação da acetil-CoA, reação mais importante na regulação da síntese de novo de ácidos graxos, e a ácido graxo sintase, que tem como produto final o palmitato. Há indícios de que dietas hipolipídicas e hiperglicídicas estimulam consideravelmente a lipogênese (28), aumentando a expressão de enzimas lipogênicas (29).

O estímulo de enzimas lipogênicas pode se dar por meio de fatores de transcrição, como o SREBP (sterol regulatory binding proteins) (30); a ChREBP (carbohydrate responsive element-binding protein), ativada em resposta à alta glicemia (28) e ao estímulo do receptor nuclear PPAR- $\gamma$ (peroxisome proliferatoractivated receptor-gamma) (31).

A própria glicose, por ser convertida a acetilCoA através da via glicolítica, estimula a lipogênese pelo fato de ser um substrato para tal processo. Além disso, a glicose plasmática estimula a lipogênese por agir no processo de liberação de insulina. A insulina é, provavelmente, o mais importante fator hormonal afetando a lipogênese, estimulando-a de maneira muito potente, aumentando a captação de glicose pelas células adiposas - via recrutamento de transportadores de glicose para a membrana plasmática - assim como ativando enzimas glicolíticas e lipogênicas (31).

Ainda se sabe muito pouco sobre a estimulação da síntese de ácidos graxos em humanos. Pode-se presumir que, em certos indivíduos, quanto mais alta a glicemia pós-prandial, maior a probabilidade de se aumentar a síntese de ácidos graxos a partir de glicose, devido ao fornecimento excessivo de átomos de carbono para o fígado $(22)$.

\section{Dieta hiperglicídica na dislipidemia}

São inúmeros os estudos que sugerem que dietas hiperglicídicas podem gerar aumento na trigliceridemia (chamado geralmente de hipertrigliceridemia induzida por carboidratos), favorecer a formação de partículas de LDL pequenas e densas e reduzir as concentrações de HDL plasmático $(4,11,22)$.

O potencial aterogênico da hipertrigliceridemia induzida por carboidratos é objeto de muito debate. Enquanto a hipertrigliceridemia observada em indivíduos seguindo dietas ricas em lipídios está mais fortemente associada a riscos aumentados de doenças cardiovasculares (32), não está claro se a hipertrigliceridemia induzida por carboidratos compartilha mecanismos semelhantes de etiologia e, portanto, se tem potencial aterogênico similar. Ou seja, diferenças na dinâmica de lipoproteínas entre as duas formas de hipertrigliceridemia podem contribuir diferentemente para o risco de doenças cardiovasculares (33).

Dois possíveis mecanismos têm sido apontados como responsáveis pelo aumento da trigliceridemia induzida por carboidratos: 1) aumento da síntese de triglicérides e conseqüente produção e liberação aumentadas de VLDL pelo fígado e 2) clearance (depuração) diminuído das partículas ricas em TG do plasma. Determinar qual destes mecanismos é responsável pela elevação da trigliceridemia é importante, uma vez que cada um deles apresenta conseqüências diversas em relação ao risco de doença cardiovascular.

Alguns autores defendem que a elevação da trigliceridemia ocorre em decorrência do aumento da produção endógena de ácidos graxos, via lipogênese de novo $(34,35)$, resultando em aumento da produção e secreção de VLDL pelo fígado. Além da lipogênese de novo, os ácidos graxos para a síntese de triglicérides (que são secretados por meio de partículas de VLDL) também podem ser derivados do pool plasmático de ácidos graxos livres, dos ácidos graxos estocados no próprio fígado e da dieta, que são disponibilizados ao fígado por meio da remoção de remanescentes de 
quilomícrons (26). Caso o aumento da secreção de partículas de VLDL seja o mecanismo responsável pelo aumento da trigliceridemia observada em conseqüência do consumo de dietas hiperglicídicas, haveria também o potencial de aumentar as concentrações de colesterol, uma vez que a secreção hepática de colesterol é proporcional à secreção de partículas de VLDL. Como as VLDL são precursoras de LDL no plasma, concentrações deste último também poderiam sofrer aumento. No entanto, alguns estudos mostram que, ao se diminuir de maneira súbita a gordura alimentar, aumentando os carboidratos sem alteração do consumo calórico total, observa-se, concomitantemente à elevação da trigliceridemia, redução das concentrações de LDL, o que pode indicar que, talvez, o aumento da produção de VLDL não seja o responsável pela elevação da trigliceridemia induzida por carboidratos. A diminuição das concentrações de LDL também dificulta concluir se a hipertrigliceridemia induzida por carboidratos realmente trará efeitos deletérios à saúde (22), uma vez que altas concentrações de LDL constituem o principal fator de risco para doença arterial coronariana $(11,24)$. De fato, a concentração de LDL é o principal alvo da terapia de redução do colesterol (24).

O outro mecanismo proposto para a elevação da trigliceridemia, a depuração reduzida de partículas ricas em triglicérides, é particularmente importante no período pós-prandial e está associado à redução nas concentrações de HDL, resultante de dietas hiperglicídicas. A depuração de partículas ricas em triglicérides pode ocorrer de duas maneiras, ambas dependentes da ação da lipase lipoprotéica (LPL), presente em tecidos extra-hepáticos, principalmente no músculo e tecido adiposo (36). A primeira é por meio do armazenamento de triglicérides, essencialmente no tecido adiposo, mas que também ocorre no músculo esquelético (na forma de triglicérides intramusculares). A segunda é a oxidação de triglicérides principalmente pelo músculo esquelético (37). Em ambos os casos, a LPL é responsável por hidrolisar os triglicérides presentes nas lipoproteínas, liberando ácidos graxos para serem utilizados pelos músculos ou armazenados nos tecidos adiposo e muscular. Quilomícrons derivados dos enterócitos competem com as partículas de VLDL (produzidas no fígado) pela remoção, promovida pelas mesmas vias lipolíticas: hidrólise de seus triglicérides pela LPL, presente no músculo esquelético e tecido adiposo (36). Isso leva a um acúmulo de lipoproteínas ricas em triglicérides (quilomícrons e VLDL) no plasma e acelera a transferência de colesterol esterificado das partículas de HDL e LDL para estas lipoproteínas ricas em triglicérides. Os triglicérides, por sua vez, são transferidos na direção oposta. Isto reduz a concentração de colesterol das partículas de HDL e gera partículas de LDL e HDL ricas em triglicérides, e depletadas em ésteres de colesterol. O conteúdo de triglicérides destas partículas é, então, hidrolisado pela lipase hepática, resultando em partículas pequenas de HDL que são removidas da circulação em um ritmo acelerado (38) e em partículas aterogênicas de LDL pequenas e densas (39). Ainda, as remanescentes de lipoproteínas ricas em triglicérides (VLDL e IDL), quando enriquecidas com colesterol, mostraram-se comprovadamente aterogênicas em animais (40). O aumento da colesterolemia é menos provável quando a hipertrigliceridemia induzida por carboidratos tem como origem a depuração reduzida de triglicérides e não a superprodução de partículas ricas em triglicérides. Outro mecanismo de depuração é a captação de lipoproteínas ricas em TG pelo figado, por meio de receptores (26). Portanto, depuração reduzida de triglicérides pode ocorrer tanto por redução da atividade da LPL quanto por falha na captação de lipoproteínas ricas em TG pelo fígado. A perda de peso e o exercício físico podem aumentar a depuração de triglicérides (33) e, portanto, podem reduzir a hipertrigliceridemia induzida por carboidratos, supondo que este mecanismo contribua para o distúrbio.

Mittendorfer \& Sidossis (4l) discordam da participação do mecanismo baseado na hipótese de depuração reduzida. Os autores submeteram indivíduos saudáveis a uma dieta hiperglicídica $(75 \%$ da energia na forma de carboidratos e $10 \%$ na forma de gorduras) e a uma dieta hiperlipídica (30\% de carboidratos e $55 \%$ de gorduras), e analisaram o metabolismo de partículas de VLDL a partir de partículas de VLDL isotopicamente marcadas. Conclui-se que a maior concentração de VLDL após a dieta hiperglicídica ocorreu em razão de maior secreção da lipoproteína e não devido à depuração aumentada. A provável explicação para a maior secreção de VLDL foi a de que seria resultado da menor oxidação de ácidos graxos derivados do plasma, observada no estudo, após dieta hiperglicídica, o que causou aumento da disponibilidade de ácidos graxos para a síntese hepática de triglicérides.

Por outro lado, Parks e cols. (33) mensuraram a contribuição dos ácidos graxos livres e da lipogênese $d e$ novo para a síntese de VLDL em homens saudáveis, antes e após 5 semanas de dieta rica em carboidratos (68\% da energia proveniente de carboidratos e 15\% proveniente de lipídios) e não encontraram evidências de que a elevação nas concentrações de triglicérides e VLDL resultasse de aumento nos níveis de ácidos graxos livres ou na taxa de lipogênese de novo. O resultado foi explicado por redução de $37 \%$ da remoção 
de TG do plasma.

Landry e cols. (42) também sugeriram que dietas ricas em carboidratos poderiam modular a trigliceridemia por meio de sua ação no clearance de triglicérides. Foi conduzido um experimento com homens saudáveis no qual uma dieta hiperglicídica $(60 \%$ do VET em carboidratos e $27 \%$ em gordura) foi comparada a uma hipoglicídica (41\% da energia proveniente de gordura e $46 \%$ de carboidratos), mas rica em ácidos graxos monoinsaturados, ambas ad libitum. Após sete semanas, os autores verificaram que a trigliceridemia diminuiu somente no grupo consumindo dieta pobre em carboidratos; indivíduos seguindo a dieta hiperglicídica não apresentaram alterações das concentrações de triglicérides. Em ambos os casos houve perda de peso (sem diferença significativa entre os grupos), mas, controlando-se a variável peso (caso o peso corporal fosse mantido constante), a dieta rica em carboidratos teria sido capaz de elevar a trigliceridemia em $16,91 \mathrm{mg} / \mathrm{dl}$. Análise multivariada mostrou que as alterações na atividade da LPL (expressa em nanomoles de ácido oléico liberados por mililitro de plasma por minuto) também foram preditoras de alterações na trigliceridemia (quanto maior a atividade da LPL, menor a concentração de TG), sendo que, após 7 semanas de intervenção nutricional, a alteração para o grupo consumindo dieta hiperglicídica foi de $-1 \pm 18$ e, para o grupo consumindo dieta hipoglicídica, de 0 $\pm 35 \mathrm{nmol} \mathrm{min-1} \mathrm{mL}^{-1}$. A oxidação de lipídios pósprandial, mensurada por meio de calorimetria indireta, foi menor em indivíduos submetidos a dieta rica em carboidratos do que submetidos a dieta hiperlipídica. Os autores sugeriram que a perda de peso observada teve efeitos benéficos sobre a trigliceridemia, compensando o seu aumento tipicamente visto em dietas isoenergéticas (com valor energético total semelhante) ricas em carboidratos. Este aumento seria modulado tanto pela alteração da oxidação de lipídios pósprandial quanto pela atividade da LPL.

Independentemente do mecanismo envolvido na elevação da trigliceridemia induzida por carboidratos, ainda se debate muito se ela é de fato fator de risco independente para doenças cardiovasculares. Estudos prospectivos têm mostrado uma relação positiva entre trigliceridemia e incidência de doenças cardiovasculares (32). No entanto, como o metabolismo lipídico é totalmente integrado, os efeitos de elevadas concentrações de triglicérides podem ser "confundidos" com aqueles decorrentes da elevação das concentrações de colesterol total e LDL ou da redução das concentrações de HDL. Além disso, a hipertrigliceridemia está freqüentemente associada a outros fatores de risco, como obe- sidade, resistência à insulina e intolerância à glicose.

Existem fortes evidências epidemiológicas da associação de baixas concentrações de HDL a doenças coronarianas (43). Embora alguns autores tenham sugerido que mudanças nas concentrações plasmáticas de HDL e triglicérides em indivíduos seguindo dietas hipolipídicas para perda de peso sejam transitórias, desaparecendo com a continuidade da dieta ou quando a eutrofia é atingida, no caso de indivíduos obesos em tratamento para perda de peso (44), estudos epidemiológicos e experimentais têm mostrado que a diminuição nos níveis de HDL pode persistir (4). No entanto, é importante ressaltar que, apesar de toda controvérsia a respeito da diminuição das concentrações de HDL resultante de dietas hipolipídicas e hiperglicídicas, nenhum estudo mostrou, até o presente, aumento na incidência de doenças cardiovasculares provocado por dietas hipolipídicas (45).

Os efeitos no perfil lipídico, mais especificamente na trigliceridemia, induzidos por dieta hiperglicídica parecem ser acentuados quando esta é rica em carboidratos de alto índice glicêmico, como é o caso de alguns monossacarídeos, alimentos processados e farinhas refinadas. Por outro lado, tais efeitos podem ser atenuados por dietas ricas em carboidratos com baixo índice glicêmico, tais como grãos integrais (11). $\mathrm{O}$ índice glicêmico é uma medida do efeito dos carboidratos dietéticos sobre a glicemia, em uma escala que varia entre 0 e 100 , sendo que valores mais altos correspondem a efeitos mais acentuados (46). Em estudo de Pawlak e cols. (47), ratos submetidos a dietas com alto IG ( $45 \%$ do VET em carboidratos, $20 \%$ proteínas e $35 \%$ de lipídios) tiveram trigliceridemia basal significativamente maior do que a de ratos alimentados submetidos a dieta com a mesma composição percentual de macronutrientes, mas com carboidratos de baixo índice glicêmico. Estudos com humanos ainda são escassos e inconclusivos (46). Apesar disso, vários órgãos de saúde, como o americano National Cholesterol Education Program (48), recomendam dietas ricas em carboidratos complexos, com baixo índice glicêmico, para pacientes com fatores de risco cardiovascular. Mais estudos são necessários nesta área a fim de dar suporte a essas recomendações.

\section{Dieta hiperglicídica e adiposidade corporal}

Um dos grandes apelos das dietas hiperglicídicas é a prevenção do ganho de peso em indivíduos eutróficos e promoção da perda de peso em indivíduos com sobrepeso (49). Isto se deve principalmente ao baixo conteúdo de lipídios deste tipo de dieta. Populações adultas consumindo dietas pobres em gorduras 
possuem baixa prevalência de obesidade $(50,51)$.

São inúmeras as desvantagens apontadas para uma dieta rica em lipídios no tratamento e prevenção do excesso de gordura corporal:

1) A gordura é o nutriente com menor capacidade de induzir saciedade e provocar diminuição da quantidade de energia consumida na refeição seguinte (52).

2) Alimentos com alta razão lipídios/ carboidratos geralmente têm maior densidade energética do que alimentos ricos em carboidratos e, portanto, promovem maior hiperfagia passiva (consumo energético maior do que o gasto, sem que haja intenção de fazê-lo), com balanço energético positivo e ganho de peso em indivíduos susceptíveis $(20,53)$. Essa hiperfagia passiva ocorre principalmente porque as pessoas tendem a ingerir o mesmo volume de alimentos, independentemente de sua composição $(52)$. Em dietas hiperlipídicas, mais calorias são consumidas passivamente. Além disso, a hiperfagia é estimulada pelo sabor e textura agradáveis conferidos pelas gorduras nos alimentos (54).

3) A gordura é mais prontamente absorvida no intestino e a perda fecal é muito menor em uma dieta com alta razão lipídios/carboidratos (20).

4) O efeito térmico dos carboidratos no processo digestório é levemente mais alto do que o dos lipídios $(6$ a $8 \%$ da energia referente aos carboidratos contra 2 a 3\% da referente aos lipídios) (55).

5) A gordura possui menor capacidade de promover auto-oxidação em razão do aumento de seu consumo $(20,52)$.

6) O excesso de energia consumida na forma de lipídios é mais eficientemente armazenado na forma de gordura corporal do que excesso calórico equivalente na forma de carboidratos (53).

A redução do conteúdo de lipídios da dieta, com conseqüente aumento da proporção de carboidratos, poderia, portanto, prevenir e tratar a obesidade. No entanto, ainda há muita controvérsia. Apesar de certos indivíduos, sob supervisão médica ou nutricional, poderem se beneficiar de dietas muito pobres em lipídios para a perda de peso, a American Heart Association (11) não as recomenda para a população em geral por diversas razões. Primeiramente, porque algumas pesquisas indicam que a perda de peso não seria sustentada por muito tempo (56). Além disso, podem levar à ingestão de ácidos graxos essenciais abaixo da recomendada e estão freqüentemente associadas ao uso de alimentos processados com teor de gordura reduzido, porém com densidade energética elevada, devido ao teor bastante aumentado de carboidratos simples. Finalmente, este tipo de dieta poderia agravar anormalidades, como baixos níveis de
HDL, hipertrigliceridemia e hiperinsulinemia (56).

Apesar de dietas ricas em carboidratos e hipolipídicas para a diminuição da adiposidade e manutenção do peso não serem consenso, diversos estudos têm mostrado resultados favoráveis a esse tipo de dieta.

Em pacientes diabéticos, Gerhard e cols. (57) compararam os efeitos de duas dietas ad libitum, uma hipolipídica/hiperglicídica e rica em fibras $(20 \%$ do VET em gorduras e $65 \%$ em carboidratos) e com menor densidade energética, e outra rica em gordura monoinsaturada ( $40 \%$ do VET em gordura, sendo $26 \%$ de monoinsaturada e $45 \%$ em carboidratos). Verificaram que houve perda de peso após 6 semanas de intervenção somente após a dieta hipolipídica rica em carboidratos e fibras. Os sujeitos tiveram ingestão energética menor $(212 \mathrm{kcal}$ a menos) quando consumiram esta dieta, quando comparada com a ingestão da dieta rica em ácidos graxos monoinsaturados. Uma vez que a densidade energética é um importante determinante da saciedade e da ingestão energética, os autores sugeriram que a menor ingestão energética durante a dieta hipolipídica estava relacionada com a menor densidade energética e, conseqüentemente, favoreceu mais a perda de peso do que a dieta rica em gordura monoinsaturada.

Estudo com indivíduos não-diabéticos com hipercolesterolemia moderada mostrou que dieta hipolipídica ad libitum (15\% de gordura referente ao VET) promove maior perda de peso do que dieta hiperlipídica (58), utilizando um desenho experimental em que cada indivíduo foi controle dele mesmo. Além disso, estudo que acompanhou por sete anos mulheres que receberam instruções para reduzir a quantidade de gordura de suas dietas, verificou que houve perda de peso no primeiro ano e que, nos anos subseqüentes, elas mantiveram peso corporal significativamente mais baixo do que mulheres do grupo controle (que não receberam tais instruções) (59). Meta-análises mostraram que a quantidade de peso perdida é proporcional à porcentagem de redução no consumo de gordura e, conseqüentemente, ao aumento da proporção de carboidratos (60).

Em animais de diferentes espécies, a correlação positiva entre adiposidade corporal e porcentagem de gordura da dieta parece estar mais estabelecida. Em estudo de Roberts e cols. (61), ratas Fisher submetidas a uma dieta hipolipídica (60\% de carboidratos, $12 \%$ de gordura e $28 \%$ de proteínas) apresentaram peso final menor do que animais submetidos a uma dieta hiperlipídica ( $40 \%$ de carboidratos, $39 \%$ de gorduras e $21 \%$ de proteínas). Boozer e cols. (62), quando compararam ratos machos Sprague-Dawley alimentados 
isoenergeticamente com rações contendo proporções crescentes de gordura (12\%, 24\%, 36\% e 48\% do VET), não encontraram diferenças significativas entre os pesos dos animais dos quatro grupos experimentais após 6 semanas de intervenção nutricional. No entanto, os autores encontraram diferenças importantes na adiposidade corporal dos animais: quanto maior a proporção de gordura da dieta, maior o depósito de gordura corporal. Ou seja, mostraram que os efeitos da gordura dietética sobre a deposição de gordura corporal ocorrem de maneira "dose-resposta", sem que haja um nível crítico abaixo do qual não há diferença entre os grupos, e que, quanto maior a porcentagem de gordura na dieta, maior a porcentagem de gordura corporal, mesmo quando a dieta é isoenergética e quando não há diferença de peso entre os grupos.

No entanto, alguns autores ainda afirmam que dietas hiperglicídicas e hipolipídicas podem ser pouco eficazes para o controle do peso (63). Segundo eles, alguns indivíduos parecem ser mais propensos ao ganho de peso por meio de dietas hiperglicídicas do que por meio de dietas hiperlipídicas. Isso porque, nestes casos, ao substituir gorduras por carboidratos, escolhem-se carboidratos com alto índice glicêmico, geralmente processados, ao invés de carboidratos complexos, como os encontrados em grãos integrais, hortaliças e diversas frutas. Mudanças hormonais e metabólicas resultantes da ingestão de dietas hiperglicídicas com alto IG são a elevação da glicemia e hiperinsulinemia pós-prandiais, que levam ao rápido armazenamento de substratos na forma de gordura corporal e estimulam a fome e a hiperfagia (63). Em estudo de Ludwig e cols. (64), verificou-se que adolescentes obesos do sexo masculino tiveram ingestão voluntária de alimentos 53\% maior após consumo de refeição com alto índice glicêmico do que com baixo IG. A rápida absorção de glicose após consumo de refeições com alto índice glicêmico induz uma série de alterações hormonais e metabólicas que podem promover consumo excessivo de alimentos.

A ingestão aguda de carboidratos promove a sua própria oxidação, diminuindo a oxidação de gordura, a qual é depositada, aumentando a adiposidade corporal $(21,63)$. Este efeito é mais acentuado quando os carboidratos possuem alto índice glicêmico. Em estudo de Pawlak e cols. (65), por exemplo, com duração de 32 semanas, animais submetidos a dietas baseadas em amidos de alto índice glicêmico apresentaram maior ganho de peso, maior aumento da adiposidade visceral e maiores concentrações de enzimas lipogênicas do que animais submetidos a dietas com o mesmo conteúdo energético, mas com índice glicêmico mais baixo (65).
Brand-Miller e cols. (63) especulam que alimentos com baixo teor de liṕ́dios e baixo índice glicêmico produzem baixa resposta glicêmica e podem auxiliar o controle do peso corporal, já que promovem a saciedade, minimizam a secreção pós-prandial de insulina e mantêm a sensibilidade à insulina.

Se, por um lado, a adoção de dietas hipolipídicas para o tratamento da obesidade ainda não é consenso, por outro, há muitas evidências de que são muito efetivas na prevenção do ganho de gordura corporal (52). Os vários tratamentos clínicos da obesidade são caracterizados por baixas taxas de sucesso e, portanto, a prevenção do ganho de peso deveria tornar-se prioridade (66).

\section{Efeito da associação à atividade física}

Os benefícios da atividade física regular para a saúde estão bem estabelecidos. $\mathrm{O}$ exercício habitual tem papel importante na manutenção da saúde global e do bem-estar (67). Evidências de estudos epidemiológicos e experimentais apontam que o exercício físico regular protege contra o desenvolvimento e a progressão de inúmeras doenças crônicas (tais como doenças cardiovasculares, hipertensão, obesidade, diabetes tipo 2, entre outras) (68), sendo, portanto, relevante componente de um estilo de vida saudável. Segundo a Organização Mundial de Saúde (9), a associação entre a prática de atividade física regular e hábitos alimentares saudáveis é a forma mais indicada de prevenção de doenças cardiovasculares, obesidade e diabetes mellitus tipo 2 .

Dentre os benefícios da atividade física regular estão a melhora do perfil lipídico, como o aumento das concentrações séricas de HDL e redução da trigliceridemia $(67,69)$, embora o exercício não tenha se mostrado eficaz no aumento das concentrações de HDL baixas isoladas, na ausência de hipertrigliceridemia (70).

Koutsari e cols. (71) procuraram verificar até que ponto a atividade física é capaz de inibir possíveis efeitos deletérios de dietas hiperglicídicas sobre a lipidemia. Testou-se a hipótese de que exercícios aeróbicos diários inibem a hipertrigliceridemia de jejum e pós-prandial observada quando há substituição de gorduras por carboidratos na dieta. Para isso, analisouse a trigliceridemia de jejum e pós-prandial (após uma refeição padrão hiperlipídica, por meio da avaliação da área abaixo da curva de trigliceridemia, com duração de 6 horas) em mulheres pós-menopausa após três situações experimentais (com 10 dias de intervalo entre elas): 1) depois de três dias de dieta hipoglicídica e hiperlipídica (35\% do VET correspondentes a carboidratos, $50 \%$ lipídios e $15 \%$ proteína); 2 ) depois de 3 dias de dieta isoenergética hiperglicídica e hipolipídica (70\% de carboidratos, $15 \%$ de lipídios e $15 \%$ de 
proteínas) e 3) após três dias da mesma dieta, porém com 60 minutos de caminhada por dia. Os valores calóricos das dietas foram ajustados para o consumo habitual de cada indivíduo. A trigliceridemia de jejum e pós-prandial foi significativamente maior após o período de consumo de dieta hiperglicídica. O exercício físico diário mostrou-se capaz de neutralizar este efeito, uma vez que não houve diferenças entre a trigliceridemia de jejum e pós-prandial após dieta hipoglicídica e dieta hiperglicídica com exercício. No entanto, os próprios autores ressaltam que a dieta hiperglicídica utilizada era rica em carboidratos simples, com maior potencial de provocar hipertrigliceridemia do que dietas ricas em carboidratos complexos.

Um dos prováveis mecanismos para a redução da trigliceridemia é o aumento da atividade da LPL induzida pela atividade física $(69,72)$, aumentando a taxa de remoção de triglicérides. Seip e cols. (73) encontraram que alterações na atividade da LPL muscular estavam inversamente correlacionadas a alterações nas concentrações de triglicérides e que há evidências de que o exercício estimula o aumento da expressão da LPL no músculo, mas não no tecido adiposo; ou seja, o exercício favorece, desta forma, a oxidação dos ácidos graxos provenientes de lipoproteínas, e não seu armazenamento. Oscai e cols. (37) verificaram que a atividade da LPL muscular em ratos exercitados é aumentada e que, portanto, o exercício tem a capacidade de remover triglicérides da circulação. Além disso, o treinamento aeróbio está associado ao aumento da capacidade de oxidação de lipídios durante exercício sub-máximo (74), o que é explicado, em parte, pelo aumento da densidade mitocondrial no músculo esquelético (75). Outra hipótese é a de que a redução da trigliceridemia resultante da prática regular de atividades físicas pode ocorrer, também, em função da redução da secreção hepática de triglicérides (76).

Diversos estudos mostram também que o exercício crônico promove melhora do metabolismo de glicose mediado pela insulina tanto em humanos quanto em animais $(77,78)$. Em modelos animais normais (não-diabéticos e sem resistência à insulina), o treinamento de intensidade de moderada a alta pode melhorar a tolerância à glicose (79), a sensibilidade à insulina (80) e aumentar o número de transportadores de glicose (GLUT-4) no músculo esquelético (81). Muitos estudos têm reportado que, após exercício crônico ou agudo, a captação muscular de glicose é maior, pois os receptores são mais responsivos à insulina, o que facilita a ressíntese dos estoques de glicogênio (82). Desta forma, dietas hiperglicídicas associadas a exercício físico crônico não acarretariam problemas em relação ao metabolismo da glicose, uma vez que todo um aparato celular e metabólico promoveria uso eficiente dos carboidratos.

A associação entre atividade física e dieta hiperglicídica e hipolipídica parece ter efeitos vantajosos sobre a composição e peso corporais, quando comparada com a associação exercício-dieta hiperlipídica. Estudo de Gleeson \& Waring (83), comparando peso e composição corporais de animais exercitados e sedentários alimentados com uma ração hipolipídica (6\% de gordura e $68 \%$ de carboidratos) e alimentados com uma ração hiperlipídica $(65 \%$ de gordura e $9 \%$ de carboidratos) mostrou que o exercício diário de natação por 60 minutos reduziu a taxa de ganho de peso em animais consumindo ração hipolipídica, porém não houve o mesmo efeito em animais consumindo ração hiperlipídica. Também foi encontrado menor teor de gordura corporal nos animais exercitados, independentemente do tipo de dieta consumido; contudo, este efeito foi muito maior no grupo que consumiu ração hipolipídica/hiperglicídica. Portanto, pode-se inferir que, embora o exercício tenha efeito de diminuir o ganho de peso, talvez este efeito possa ser reduzido no caso de consumo maior de gorduras (dieta hiperlipídica).

Dietas hiperglicídicas e hipolipídicas mostramse adequadas a atletas, que têm como objetivo a melhora da performance, uma vez que promovem maiores estoques de glicogênio muscular (82) e, conseqüentemente, maior tolerância ao esforço, já que é sabido há tempos que a concentração de glicogênio muscular é um fator determinante para o desempenho no exercício aeróbio moderado e prolongado (84). Guias dietéticos para atletas são unânimes em recomendar consumo de dietas ricas em carboidratos (85).

No entanto, nota-se que existem muitos estudos relacionando dieta ao desempenho de atletas, mas poucos procuram associar a atividade física e padrões alimentares à saúde da população geral. Recomendações para adoção de dietas pobres em gordura têm atingido não somente atletas, mas indivíduos fisicamente ativos, que não têm necessariamente a alta performance como meta. Além disso, as recomendações para diminuição do consumo de lipídios têm também atingido a população em geral, inclusive indivíduos sedentários. No entanto, alguns autores sustentam que, na ausência de uma vida fisicamente ativa, a ingestão crônica de dietas hiperglicídicas pode prejudicar a sensibilidade do músculo esquelético à insulina, à liponeogênese a partir de carboidratos e ao aumento da produção hepática de VLDL e, por isso, não recomendam dietas hiperglicídicas a indivíduos sedentários (21). 


\section{CONSIDERAÇŌES FINAIS}

Apesar das inúmeras recomendações existentes, ainda há muita controvérsia a respeito de qual seria a composição ideal da dieta para a população em geral, a fim de promover a saúde e evitar problemas tais como o aumento da adiposidade e dislipidemias. Enquanto os efeitos deletérios de dietas hiperlipídicas já estão bem estabelecidos, dietas ricas em carboidratos têm sido largamente recomendadas na tentativa de se diminuir o teor de gordura da dieta, partindo de recomendações de órgãos oficiais de saúde de diversos países (7-11), incluindo o Brasil (9). Vários estudos apontam para possíveis efeitos deletérios de se adotar dietas hiperglicídicas/hipolipídicas e ainda não se sabe a extensão com que a atividade física pode minimizar esses efeitos em longo prazo e quais os mecanismos envolvidos nesse processo.

Estudos epidemiológicos têm demonstrado que, apesar de atacadas por alguns autores, dietas hipolipídicas e ricas em carboidratos e fibras apresentam maior capacidade de promover a longevidade e a qualidade de vida $(10,86)$. Não são encontrados estudos que tenham descoberto associação positiva entre tais parâmetros e dietas ricas em lipídios.

Futuros estudos a respeito da composição ideal da dieta devem enfocar a qualidade de carboidratos e gorduras. $\mathrm{O}$ estudo dos efeitos do índice glicêmico dos alimentos, por exemplo, pode fornecer dados importantes a respeito da relação entre alimentação e doenças crônicas (87).

Investigações sobre a proporção entre diferentes tipos de lipídios (monoinsaturados, poliinsaturados e saturados) já apontam resultados interessantes. Em estudo feito no Sri Lanka (88), mostrou-se que, apesar de o consumo médio de gordura da população ser de $25 \%$ do VET (e, portanto, de acordo com a maioria das recomendações dietéticas), as estimativas de mortes por doenças cardiovasculares são maiores do que as encontradas em muitos países ocidentais. Descobriu-se que a razão entre ácidos graxos saturados e ácidos graxos poliinsaturados consumidos era de 9 para 1 , ou seja, muito maior do que a razão recomendada pela American Heart Association (89), de l para 1. Pesquisadores sugerem que o consumo relativamente baixo em gorduras poliinsaturadas, em associação com o consumo relativamente alto de carboidratos (65\% do VET), parece resultar em hipertrigliceridemia e baixas concentrações de HDL e predispor a eventos cardiovasculares. Ou seja, é provável que a dieta desta população, que estava de acordo com a maioria das recomendações, não tenha sido suficiente para prevenir doenças cardiovasculares devido à grande inadequação entre os tipos de lipídios consumidos.

É importante lembrar que muitas das críticas feitas às recomendações de dietas hipolipídicas têm como base a pouca ênfase que se dá ao consumo de quantidades adequadas de fibras (na forma de grãos integrais e vegetais), de frutas e hortaliças, além da substituição de parte da gordura saturada por gorduras poli e monoinsaturadas. Mais esforços devem ser feitos no sentido de se instruir a população não só em relação às proporções de macronutrientes mais adequadas mas, também, à qualidade dos mesmos. Embora grande parte das recomendações atuais já inclua tópicos neste sentido, ainda há muito que se avançar nesta área, tanto no âmbito da educação da população quanto em pesquisas aplicadas.

\section{AGRADECIMENTOS}

Os autores agradecem a Fernanda Baeza Scagliusi pela revisão do texto e pelas sugestões oferecidas, e ao apoio concedido pela FAPESP (processo 02/11247-2).

\section{REFERÊNCIAS}

1. Dawber TR, Moore FE, Mann GV. Coronary heart disease in the Framingham Study. Am J Publ Health 1957;47:4-24.

2. Keys A, Anderson JT, Grande F. Prediction of serum-cholesterol responses of man to changes in fats in the diet. Lancet 1957;l:943-66.

3. Taubes G. The soft science of dietary fat. Science $2001 ; 291: 2536-45$.

4. Katan MB. Effect of low-fat diets on plasma high-density lipoprotein concentrations. Am J Clin Nutr 1998;67 (suppl):573-6.

5. Mokdad AH, Serdula MK, Dietz WH, Bowman BA, Marks JS, Koplan JP. The spread of the obesity in the United States, 1991-1998. JAMA 1999;282:1519-22.

6. Mendez MA, Monteiro CA, Popkin BM. Overweight exceeds underweight among women in most developing countries. Am J Clin Nutr 2005;81:714-21.

7. USDA (U.S. Department of Agriculture). Food Guide Pyramid. Home and Garden Bulletin 252. August, 1992.

8. U.S. Department of Health and Human Services and U.S. Department of Agriculture. Dietary Guidelines for Americans 2005. $6^{\text {th }}$ ed. Washington, DC: U.S. Government Printing Office, January, 2005.

9. Brasil. Ministério da Saúde. Secretaria de Atenção à Saúde. Coordenação-Geral da Política de Alimentação e Nutrição. Guia alimentar para a população brasileira: Promovendo a alimentação saudável / Ministério da Saúde, Secretaria de Atenção à Saúde, Coordenação-Geral da Política de Alimentação e Nutrição - Brasília: Ministério da Saúde, 2005.

10. WHO (World Health Organization). Diet, nutrition and the prevention of chronic diseases. WHO Technical Report Series 916. Geneva, 2003.

11. Krauss RM, Eckel RH, Howard BV, Appel LJ, Daniels SR, Deckelbaum RJ, et al. AHA Dietary Guidelines. Revision 2000: a statement for healthcare professionals from the Nutrition Committee of the American Heart Association. Circulation 2000;102:2284-99. 
12. Ornish D. Low-fat diets. N Engl J Med 1998;338:128.

13. Scagliusi FB, Polacow VO, Artioli GG, Benatti FB, Lancha Jr $\mathrm{AH}$. Selective underreporting of energy intake in women: Magnitude, determinants, and effect of training. J Am Diet Assoc 2003;103(10):1306-13.

14. Goris AHC, Westerterp-Olatenga MS, Westerterp KR. Undereating and underrecording of habitual food intake in obese men: selective underreporting of fat intake. Am J Clin Nutr 2000;71:130-4.

15. Willet WC. Dietary fat plays a major role in obesity: no. Obes Rev 2002;3:59-68.

16. Bray GA, Popkin BM. Dietary fat intake does affect obesity! Am J Clin Nutr 1998;68:1157-73.

17. Allison DB, Saunders SE. Obesity in North America, an overview. Med Clin North Am 2000;84:305-32.

18. Rubenstein AH. Obesity: a modern epidemic. Trans Am Clin Climatol Assoc 2005;116:103-11.

19. Bazzano LA, Serdula M, Liu S. Prevention of type 2 diabetes by diet and lifestyle modification. J Am Coll Nutr 2005;24(5):310-9.

20. Astrup $A$. The role of dietary fat in the prevention and treatment of obesity. Efficacy and safety of low-fat diets. Int J Obes Relat Metab Disord 2001;25(suppl 1):S46-50.

21. Graham TE, Adamo KB. Dietary carbohydrate and its effects on metabolism and substrate stores in sedentary and active individuals. Can J Appl Physiol 1999;24(5):393-415.

22. Parks EJ. Effect of dietary carbohydrate on triglyceride metabolism in humans. J Nutr 2001;131:2772S-4.

23. Grundy SM, Abate N, Chandalia M. Diet composition and the metabolic syndrome: What is the optimal fat intake? Am J Med 2002;113(9B):25S-9.

24. National Institutes of Health. National Heart, Lung and Blood Institute. Third Report of the National Cholesterol Education Program (NCEP). Expert Panel on Detection, Evaluation, and Treatment of High Blood Cholesterol in Adults (Adult Treatment Panel III). NIH Publication No 02-5115; 2002.

25. Estruch R, Martinez-Gonzalez MA, Corella D, Salas-Salvado J, Ruiz-Gutierrez V, Covas MI, et al. Effects of a Mediterraneanstyle diet on cardiovascular risk factors: a randomized trial. Ann Intern Med 2006;145(1):1-11.

26. Parks EJ. Dietary carbohydrate's effects on lipogenesis and the relationship of lipogenesis to blood insulin and glucose concentrations. Br J Nutr 2002;87(suppl 2):S247-53

27. Hellerstein MK. Methods for measurement of fatty acid and cholesterol metabolism. Curr Opin Lipidol 19956(3):172-81.

28. Uyeda K, Yamashita H, Kawaguchi T. Carbohydrate responsive element-binding protein (ChREBP): a key regulator of glucose metabolism and fat storage. Biochem Pharmacol 2002;63:2075-80.

29. Delzenne N, Ferre P, Beylot M, Daubioul C, Declerq B, Diraison $F$, et al. Study of the regulation by nutrients of the expression of genes involved in lipogenesis and obesity in humans and animals. Nutr Metab Cardiovasc Dis 2001;11/4 suppl):118-21.

30. Stoeckman AK, Towle HC. The role of SREBP-1c in nutritional regulation of lipogenic enzyme gene expression. J Biol Chem 2002;277(30):27029-35.

31. Kersten S. Mechanisms of nutritional and hormonal regulation of lipogenesis. EMBO reports 2001;21(41):282-6.

32. Austin MA, Holkanson JE, Edwards KL. Hypertriglyceridemia as a cardiovascular risk factor. Am J Cardiol 1998;81:7B-12.

33. Parks EJ, Krauss RM, Christiansen MP, Neese RA, Hellerstein MK. Effects of a low-fat, high-carbohydrate diet on VLDLtriglyceride assembly, production and clearance. J Clin Invest 1999;104:1087-96.

34. Nestel PJ, Carroll KF, Havenstein N. Plasma triglyceride response to carbohydrates, fats and calorie intake. MetaboIism 1970;19:1-18.

35. Boberg J, Carlson LA, Freyschuss U, Lassers BW, Wahlqvist ML. Splanchnic secretion rates of plasma triglycerides and total and splanchnic turnover of plasma free fatty acids in men with normo- and hypertriglyceridaemia. Eur J Clin Invest 1972;2:454-66.
36. Björkegren J, Packard CJ, Hamsten A, Bedford D, Caslake M, Foster $\mathrm{L}$, et al. Accumulation of large very low density lipoprotein in plasma during intravenous infusion of a chylomicron-like triglyceride emulsion reflects competition for a common lipolytic pathway. J Lip Res 1996;37:76-86.

37. Oscai LB, Tsika RW, Essig DA. Exercise training has a heparin-like effect on lipoprotein lipase activity in muscle. Can J Physiol Pharmacol 1992;70:905-9.

38. Rashid S, Uffelman KD, Lewis GF. The mechanism of HDL lowering in hypertriglyceridemic, insulin-resistant states. J Diabetes Complicat 2002;16:24-8.

39. Griffin BA. Low-density lipoprotein subclasses: mechanisms of formation and modulation. Proc Nutr Soc 1997;56:693-702.

40. Breslow JL. Mouse models of atherosclerosis. Science 1996;272:685-8.

41. Mittendorfer B, Sidossis LS. Mechanism for the increase in plasma triacylglycerol concentrations after consumption of short-term, high carbohydrate diets. Am J Clin Nutr 2001;73:892-9.

42. Landry N, Bergeron N, Archer R, Samson P, Corneau L, Bergeron $\mathrm{J}$, et al. Whole-body fat oxidation rate and plasma triacylglycerol concentrations in men consuming an ad libitum high-carbohydrate or low-carbohydrate diet. Am J Clin Nutr 2003;77:580-6.

43. Despres JP, Lemieux I, Dagenais GR, Cantin B, Lamarche B. $\mathrm{HDL}$-cholesterol as a marker of coronary heart disease risk: the Quebec cardiovascular study. Atherosclerosis 2000;153(2):263-72

44. Nonas CA. A model for chronic care of obesity through dietary treatment. J Am Diet Assoc 1998;98(suppl 2):s16-22.

45. Mensink RP, Katan MB. Effect of dietary fatty acids on serum lipids and lipoproteins. A meta-analysis of 27 trials. Arterioscler Thromb 1992;12:911-9.

46. Kripke C. Does a low glycemic index diet reduce $\mathrm{CHD}$ ? Am Fam Physician 2005;72(7):1224.

47. Pawlak DB, Bryson JM, Denyer GS, Brand-Miller JC. High glycemic index starch promotes hypersercretion of insulin and higher body fat in rats without affecting insulin sensitivity. J Nutr 2001;131:99-104.

48. National Institutes of Health (NIH). National Heart, Lung and Blood Institute. Third Report of the National Cholesterol Education Program (NCEP). Expert Panel on Detection, Evaluation, and Treatment of High Blood Cholesterol in Adults (Adult Treatment Panel III). NIH Publication No 025115; 2002. Disponível em <http://www.nhlbi.nih.gov/guidelines/cholesterol/atp3_rpt.htm>. Acessado em: 15 maio 2005.

49. Kennedy ET, Bowman SA, Spence JT, Freedman M, King J. Popular diets: correlation to health, nutrition and obesity. J Am Diet Assoc 2001;101:411-20.

50. Popkin BM, Ge K, Zhai Z, Guo X, Ma H, Zohoori N. The nutrition transition in China: a cross-sectional analysis. Eur J Clin Nutr 1993;47:333-46.

51. Monteiro $C$, Mondini L, de Souza ALM, Popkin BM. The nutrition transition in Brazil. Eur J Clin Nutr 1995;49:105-13.

52. Prentice AM. Manipulation of dietary fat and energy density and subsequent effects on substrate flux on food intake. Am J Clin Nutr 1998;67(suppl):535s-41.

53. Kirk TR. Role of dietary carbohydrate and frequent eating in body-weight control. Proc Nutr Soc 2000;59:349-58.

54. Drewnowski A, Kurth C, Holden-Wiltse J, Saari J. Food preferences in human obesity: carbohydrates versus fats. Appetite 1992;18:207-21.

55. Thiébaud D, Schutz Y, Acheson K, Jacot E, Defronzo RA, Felber JP, et al. Energy cost of glucose storage in human subjects during glucose-insulin infusions. Am J Physiol 1983;244:E216-21.

56. Kasim-Karakas SE, Almario RU, Mueller WM. Changes in plasma lipoproteins during low-fat, high-carbohydrate diets: effects of energy intake. Am J Clin Nutr 2000;71:1439-47.

57. Gerhard GT, Ahmann A, Meeuws K, McMurry MP, Duell PB, Connor WE. Effects of a low-fat diet compared with those of a high-monounsaturated fat diet on body weight, plasma lipids and lipoproteins, and glycemic control in type 2 diabetes. Am J Clin Nutr 2004;80:668-73. 
58. Schaefer EJ, Lichtenstein AH, Lamon-Fava S, McNamara JR, Schaefer MM, Rasmussen $\mathrm{H}$, et al. Body weight and low-density lipoprotein cholesterol changes after consumption of a low-fat ad libitum diet. JAMA 1995;274:1450-5.

59. Howard BV, Manson JE, Stefanick ML, Beresford SA, Frank G, Jones $B$, et al. Low-fat dietary pattern and weight change over 7 years: the Women's Health Initiative Dietary Modification Trial. JAMA 2006;295(1):39-49.

60. Astrup A, Ryan L, Grunwald GK, Storgaard M, Saris W, Melanson $\mathrm{E}$, et al. The role of dietary fat in body fatness: evidence from a preliminary meta-analysis of ad libitum low-fat dietary intervention studies. J Nutr 2000;83(suppl 1):s25-32.

61. Roberts CK, Barnard RJ, Liang KH, Vaziri ND. Effect of diet on adipose tissue and skeletal muscle VLDL receptor and LPL: implications for obesity and hyperlipidemia. Atherosclerosis 2002;16:133-41.

62. Boozer C, Schoenbach G, Atkinson RL. Dietary fat and adiposity: a dose-response relationship in adult male rats fed isocalorically. Am J Physiol 1995;268(Endocrinol Metab 31):E546-50.

63. Brand-Miller JC, Holt SHA, Pawlak DB, McMillan J. Glycemic index and obesity. Am J Clin Nutr 2002;76(suppl):281S-5.

64. Ludwig DS, Majzoub JA, Al-Zahrani A, Dallal GE, Blanco Roberts SB. High glycemic index foods, overeating, and obesity. Pediatrics 1999;103:E26.

65. Pawlak D, Denyer GS, Brand-Miller JC. Long-term feeding with high glycemic index starch leads to obesity in mature rats. Proc Nutr Soc Aust 2000;24:215

66. National Task Force on Prevention and Treatment of Obesity. Towards prevention of obesity: research directions. Obes Res 1994:2(6):571-84

67. ACSM (American College of Sports Medicine). Benefícios e riscos associados aos exercícios. In: Teste de esforço e Prescrição de Exercício. 5a ed. Rio de Janeiro: Revinter, 2000. pp. 3-10.

68. Ketelhut RG, Franz IW, Sholze J. Regular exercise as an effective approach in antihypertensive therapy. Med Sci Sports Exerc 2004;36:4-8.

69. Kantor MA, Cullinane EM, Sady SP, Herbert PN, Thompson PD. Exercise acutely increases HDL-cholesterol and lipoprotein lipase activity in trained and untrained men. Metabolism 1987;36:188-92.

70. Thompson PD, Rader DJ. Does exercise increase HDL cholesterol in those who need it the most? Arterioscler Thromb Vasc Biol 2001;21:1097-8.

71. Koutsari C, Karpe F, Humphreys SM, Frayn KN, Hardman AE. Exercise prevents the accumulation of triglyceride-rich lipoprotein and their remnants seen when changing to a high-carbohydrate diet. Arterioscler Thromb Vasc Biol 2001;21:1520-5.

72. Herd SL, Kiens B, Boobis LH, Hardman AE. Moderate exercise, postprandial lipemia, and skeletal muscle lipoprotein lipase activity. Metabolism 2001:50(7):756-62

73. Seip RL, Angelopoulos TJ, Semenkovich CF. Exercise induces human lipoprotein lipase gene expression in skeletal muscle but not adipose tissue. Am J Physiol 1995;272:E229-36.

74. Jansson E, Kaijser L. Substrate utilization and enzymes in skeletal muscle of extremely endurance-trained men. J Appl Physiol 1987;62(3):999-1005.

75. Holloszy JO. Biochemical adaptations in muscle. Effects of exercise on mitochondrial oxygen uptake and respiratory enzyme activity in skeletal muscle. J Biol Chem $1967 ; 242(9): 2278-82$.
76. Zavaroni I, Chen YI, Mondon CE, Reaven GM. Ability of exercise to inhibit carbohydrate-induced hypertrigliceridemia in rats. Metabolism 1981;30(5):476-80.

77. Goodyear LJ, Kahn BB. Exercise, glucose transport, and insulin sensitivity. Annu Rev Med 1998;49:235-61.

78. Hayashi T, Wojtaszewski JF, Goodyear LJ. Exercise regulation of glucose transport in skeletal muscle. Am J Physiol Endocrinol Metab 1997;273:E1039-51.

79. Berger M, Kemmer FW, Becker K, Herberg L, Schwenen M, Gjinavci $A$, et al. Effect of physical training on glucose tolerance and on glucose metabolism of skeletal muscle in anaesthetized normal rats. Diabetologia 1979;16(3):179-84.

80. James DE, Kraegen EW, Chisholm DJ. Effects of exercise training on in vivo insulin action in individual tissues of the rat. J Clin Invest 1985;76:657-66.

81. Henriksen EJ, Halseth AE. Early alterations in soleus GLUT-4, glucose transport, and glycogen in voluntary running rats. $\mathbf{J}$ Appl Physiol 1994:76(5):1862-7.

82. Pereira LO, Lancha Jr AH. Effect of insulin and contraction up on glucose transport in skeletal muscle. Prog Biophys Mol Biol 2004;84:1-27.

83. Gleeson M, Waring JJ. Influence of diet on the storage, mobilization and utilization of energy reserves in trained and untrained rats. Comp Biochem Physiol 1986;85A(3):411-5.

84. Bergstrom J, Hermansen L, Hultman E, Saltin B. Diet, muscle glycogen and physical performance. Acta Physiol Scand 1967:71:140-50

85. Burke LM, Cox GR, Culmmings NK, Desbrow B. Guidelines for daily carbohydrate intake: do athletes achieve them? Sports Med 2001;31:267-99.

86. Diehr $P$, Beresford SA. The relation of dietary patterns to future survival, health, and cardiovascular events in older adults. J Clin Epidemiol 2003;56(12):1224-35.

87. Brand-Miller JC. Glycemic load and chronic disease. Nutr Rev 2003;61(5):S56-60.

88. Abeywardena MY. Dietary fats, carbohydrates and vascular disease: Sri Lankan perspectives. Atherosclerosis 2003;171(2):157-61.

89. Kris-Etherton $\mathrm{P}$, Daniels SR, Eckel RH, Engler M, Howard BV, Krauss RM, et al. Summary of the scientific conference on dietary fatty acids and cardiovascular health: conference summary from the nutrition committee of the American Heart Association. Circulation 2001;103(7):1034-9.

\section{Endereço para correspondência:}

Viviane Ozores Polacow

Departamento de Biodinâmica

Escola de Educação Física e Esporte - USP

Laboratório de Nutrição e Metabolismo Aplicados à Atividade Motora

Av. Professor Mello Moraes 65

05508-900 São Paulo, SP

Fax: (11) 3813-5921

E-mail: vpolacow@usp.br 